Martin Höpner und Bojan Jurczyk

\title{
Kritik des Eurobarometers
}

Über die Verwischung der Grenze zwischen seriöser Demoskopie und interessengeleiteter Propaganda

»Kurz vor der Erweiterung ... und in einem Klima der Verständnislosigkeit gegenüber dem Globalisierungsprozess ist es notwendiger denn je, dass das europäische Projekt für sinnvoll gehalten wird ...«1

\section{Einleitung}

Zahlreiche politikwissenschaftliche Erkenntnisse über die integrationspolitischen Einstellungen der europäischen Bürgerinnen und Bürger beruhen auf Daten des Eurobarometers. ${ }^{2}$ Dabei handelt es sich um eine Serie von Befragungen, die seit 1973 zweimal jährlich in allen Mitgliedsländern der EWG/EG/EU (nachfolgend der Einfachheit halber stets: der EU) durchgeführt wird. Eine besondere Stärke des Eurobarometers besteht darin, dass den Befragten neben stets wechselnden Spezialfragen auch eine Anzahl an Standardfragen gestellt wird, die über längere Perioden beibehalten werden. Die aus den Umfragen gewonnenen Datensätze erlauben deshalb nicht nur Länder-, sondern auch Zeitvergleiche und sind aufgrund ihrer Reichhaltigkeit zur Klärung einer Vielzahl von Fragestellungen nutzbar. Ergänzt wird das Standard-Eurobarometer um zusätzliche Erhebungswellen zu wechselnden Schwerpunkthemen (das sogenannte Flash Eurobarometer).

Die Kernfragen des Eurobarometers betreffen das Meinungsbild der Bürgerinnen und Bürger gegenüber den europäischen Institutionen, der europäischen Politik und dem Integrationsprozess insgesamt. Darüber hinaus werden Einstellungsdaten beispielsweise zur sozialen Situation in den Mitgliedsstaaten und zu Politikbereichen wie der Außen-, Wirtschafts-, Sicherheits- und Kulturpolitik erhoben, um nur einige zu nennen. Die Daten werden durch persönlich-mündliche Interviews (CAPI) gewonnen. Pro Mitgliedsstaat werden um die 1.000 Personen ab dem Alter von 15 Jahren befragt, wobei die genaue Anzahl entlang der Ländergröße variiert. So

1 Aus der Mitteilung der Europäischen Kommission zur Informations- und Kommunikationsstrategie der EU, KOM/2002/0350 endg.

2 Einen aktuellen Überblick bieten Tiemann et al. 2011. 
beträgt die Anzahl der interviewten Personen rund 1.500 im Falle Deutschlands und rund 500 im Falle Luxemburgs, Zyperns und Maltas. ${ }^{3}$

Wir wollen nicht bestreiten, dass die Daten des Eurobarometers von der Integrationsforschung sinnvoll genutzt werden können. Gleichwohl besteht ein erhebliches Problem, das stets genau dann an Brisanz gewinnt, wenn es um das eigentliche Kernanliegen des Eurobarometers geht: um Erkenntnisse über die Integrationspräferenzen der Bürgerinnen und Bürger. Zur Klärung dieser Fragestellung ist die Auftraggeberin der Eurobarometer-Umfragen denkbar schlecht geeignet. Denn hierbei handelt es sich um die Kommission, deren Politik selbst Gegenstand der Befragungen ist. Sie steht den Haltungen zur europäischen Integration nicht neutral gegenüber. Vielmehr sind bestimmte Ergebnisse zur legitimatorischen Unterfütterung ihrer Politik geeigneter als andere. Sie ist daran interessiert, den Integrationswillen der Bürgerinnen und Bürger so ausgeprägt wie nur möglich darstellen zu können. Die Kommission sieht sich folglich mit dem Anreiz konfrontiert, die Grenze zwischen wissenschaftlich redlicher Befragung und manipulativer Propaganda zu verwischen. Dass sie diesem Anreiz tatsächlich nachgibt, ist Gegenstand unseres Beitrags.

Im Einzelnen argumentieren wir, dass der Mangel an demokratischer Legitimation europäischer Politik aus strukturellen Gründen fortbesteht. Die Behebung dieses Mangels liegt nicht in der Hand der Kommission. Umso mehr ist die Kommission bestrebt, den Nachweis zu führen, dass ihre Integrationspolitik im Einklang mit den Präferenzen der Bürgerinnen und Bürger steht. Eines der wichtigsten ihr hierbei zur Verfügung stehenden Instrumente ist das Eurobarometer. Die EurobarometerDaten sind somit kein bloßes "statistisches Material « zur Nutzung in den Elfenbeintürmen der Wissenschaft, sondern ein hochpolitisches Instrument zur (vermeintlichen) Rechtfertigung von Integrationspolitik. Wie insbesondere Gramberger ${ }^{4}$ herausgearbeitet hat, wurde das Eurobarometer bis zum Ende der Ära Delors in der Tat strategisch zur Allokation von Scheinlegitimation eingesetzt. ${ }^{5} \mathrm{Im}$ empirischen Teil dieses Beitrags wenden wir uns daher der Ära nach Delors zu und zeigen, dass manipulative Tendenzen des Eurobarometers bis in die jüngste Vergangenheit hinein fortbestehen.

Unser Ziel besteht darin, den Blick für die Probleme der wissenschaftlichen Brauchbarkeit der Eurobarometer-Befragungen zu schärfen. Wer EurobarometerDaten für eigene Analysen nutzt oder sich anhand der Kommissionsberichte über die Haltungen der europäischen Bürgerinnen und Bürger informiert, sollte sich der Verwischungen der Grenze zwischen Demoskopie und Meinungsmache bewusst

3 Diese Angaben beziehen sich auf die Standard-Eurobarometer-Berichte 73 und 74 aus dem Jahr 2010, wobei die Anzahl der deutschen Probanden im Eurobarometer 74 mit rund 1.600 etwas höher lag.

4 Gramberger 1997.

5 Vgl. für diese Phase insbesondere die Kritik von Noelle-Neumann 1993, S. 26-34. Namentlich kritisierte sie die zum Einsatz kommenden »Sonnenscheinfragen «, also »Frageformulierungen, die nur die positive, nicht die negative Seite der Reaktion der öffentlichen Meinung erkennen lassen«; ebd., S. 27. 
sein. Und die seriöse Forschung sollte sich deutlicher als bisher von den Praktiken des Eurobarometers abgrenzen.

\section{Knappe Legitimitätsressourcen europäischer Entscheidungen}

Je stärker europäische Entscheidungen in die Lebenswelt der Bürgerinnen und Bürger eingreifen, umso mehr wächst der Bedarf, die europäische Politik legitimatorisch zu unterfüttern. Zum Verständnis der Neigung der Kommission, Scheinlegitimitäten zu erzeugen, ist die von Fritz W. Scharpf eingeführte Unterscheidung zwischen Input- und Output-Legitimität hilfreich: Input-Legitimität wird demnach erreicht durch demokratische Verfahrensweisen, mit der eine Regierungsinstitution arbeitet, Output-Legitimität dagegen bemisst sich allein am Erfolg einer Politik. ${ }^{6}$ Wir wenden uns zunächst der Input-Seite zu.

In der Frühphase des europäischen Integrationsprozesses verfügte das Europäische Parlament (EP) lediglich über schwache Rechte, und erst seit 1979 wird es von den europäischen Bevölkerungen direkt gewählt. Gleichwohl wurde in dieser Phase nur hin und wieder ein europäisches Demokratiedefizit beklagt. Dies lag zum einen daran, dass europäische Politik nur selten in politisch stark hervorgehobene Bereiche eindrang. Zum anderen bot das Prinzip der Einstimmigkeit im Rat Schutz vor Verletzungen demokratischer Selbstbestimmung. Denn wo immer eine geplante europäische Entscheidung den Interessen einer demokratisch legitimierten nationalen Regierung zuwiderlief, konnte sie dafür sorgen, dass der Status quo erhalten blieb.

Seit Verabschiedung der Einheitlichen Europäischen Akte im Jahr 1986 ging der Rat zunehmend zu Mehrheitsentscheidungen über. Spätestens mit diesem Schritt konnte sich das Prinzip der abgeleiteten - also über die demokratischen Prozesse in den Mitgliedsstaaten hergestellten - Legitimation nicht mehr als hinreichend erweisen. Denn nunmehr konnten europäische Entscheidungen den Interessen eines Mitgliedslands fundamental zuwiderlaufen, dennoch kamen sie zustande und mussten in inländisches Recht überführt werden. Um dies vom Verfahren her zu legitimieren, müsste das europäische politische System eine eigene Demokratiequalität aufweisen.

Nach vorherrschender Ansicht hat sich diese Legitimationslücke bis heute nicht geschlossen. ${ }^{7}$ Umstritten ist, ob sie im Zeitverlauf kleiner oder größer geworden ist. Für eine Verkleinerung der Lücke spricht, dass die Rechte des EP - insbesondere

6 Scharpf 1999, Kapitel 1.

7 Siehe Follesdal, Hix 2006; Neyer 2011. Einen Überblick über unterschiedliche Einschätzungen zum europäischen Demokratiedefizit bietet Schäfer 2006. Er unterscheidet Optimisten (die das Defizit an Input-Legitimation für institutionell behebbar halten), Pessimisten (die eine solche Behebung für mittelfristig ausgeschlossen halten), Apologeten (die eine Demokratisierung für möglich, aber nicht wünschenswert halten) und Fatalisten (die eine Demokratisierung weder für möglich halten noch sie erstrebenswert finden). Selten ist hingegen, dass die Legitimationslücke (bezogen auf die Input-Seite des politischen Prozesses) rundum geleugnet wird; siehe etwa Moravcsik 2008. 
durch den Vertrag von Lissabon - zweifellos gestärkt wurden. ${ }^{8}$ Für eine Vergrößerung der Lücke spricht hingegen, dass die Vertiefung der Union und damit der Bedarf an Legitimation schneller wächst als die Demokratisierung des politischen Systems der EU und dass sich die EU mit jeder Erweiterungsrunde weiter von den "vor-institutionellen " Voraussetzungen einer europäischen Demokratie entfernen könnte: einer europäischen Öffentlichkeit und einem gemeinsamen »Wir-Gefühl «, das europäische Entscheidungen auch dann legitimieren könnte, wenn der vertiefte Integrationsprozess nicht nur Gewinner, sondern auch Verlierer hervorbringt. ${ }^{9}$

Der Mangel an Legitimation durch demokratische Verfahren beruht allerdings nicht nur auf den demokratischen Defiziten der europäischen Politik, sondern auch darauf, dass ein beträchtlicher Anteil eigentlich legitimationsbedürftiger Entscheidungen gar nicht erst von den politischen Organen der Mitgliedsländer oder der EU erfasst werden. Gemeint ist hier die sogenannte »Integration durch Recht", also jener Teil der Integration, der nicht durch politische Übereinkünfte zwischen den Mitgliedsstaaten, sondern durch die extensive Interpretation des europäischen Rechts durch die supranationalen Organe Kommission und Europäischer Gerichtshof $(\mathrm{EuGH})$ zustande kommt. ${ }^{10}$ Wichtige Ergebnisse der »Integration durch Recht " sind der Vorrang des Europarechts gegenüber den nationalen Rechtsordnungen und seine Direktwirkung, die Behandlung der Grundfreiheiten als grundrechtsähnliche Individualrechte, die Schöpfung europäischer Grundrechte, die horizontale Drittwirkung europäischer Individualrechte auf Private und eine stetige Ausdehnung des europäischen Wettbewerbsrechts sowie der Grundfreiheiten auf neue Sektoren und neue, zuvor der Autonomie der Mitgliedsstaaten vorbehaltene Regelungsbereiche. $\mathrm{Ob}$ diese Entwicklungen möglich wurden, weil sie stets unter "stillschweigender Billigung « der mächtigsten Mitgliedsstaaten erfolgten (so die Sicht der Intergouvernementalisten), oder ob sie darauf hindeuten, dass sich die supranationalen Organe autonome Spielräume erkämpft haben, die Integration auch gegen die Interessen der Mitgliedsstaaten voranzutreiben (so die Sicht der Supranationalisten), ${ }^{11}$ macht für die Zwecke der hier geführten Argumentation keinen Unterschied: In beiden Fällen fallen Integrationsentscheidungen zunächst einmal außer Reichweite der demokratisch legitimierten Organe und vergrößern deshalb das Legitimitätsdefizit europäischen Regierens.

Halten wir fest: Die Legitimation der EU durch demokratische Verfahren erweist sich als defizitär. Scharpfs Lehre von den zwei Formen der Legitimation zufolge muss die Akzeptanz von Entscheidungen nicht immer aus ihrer demokratischen Rückbindung, sondern kann auch aus der Übereinstimmung ihrer Folgen mit den Präferenzen der Regierten resultieren, also: aus ihrem » Output «. Ein Beispiel hierfür sind die geldpolitischen Entscheidungen autonomer Zentralbanken, die bewusst

\section{Oeter 2009.}

9 Höpner, Schäfer 2012.

10 Alter 2001, 2009; Höpner, Schäfer 2010; Scharpf 2009.

11 Vergleiche jüngst die Debatte zwischen Carruba et al. 2008 und Stone Sweet/Brunell 2012. 
dem Zugriff der demokratisch legitimierten Politik entzogen wurden und gleichwohl als legitim empfunden werden, weil sie nach vorherrschender Sicht besser in der Lage sind, befriedigende Ergebnisse zu erzeugen. Gerade also in Anbetracht der demokratischen Defizite europäischen Regierens könnte der Integrationsprozess von dem Nachweis profitieren, im Einklang mit den Integrationspräferenzen der Bürgerinnen und Bürger zu stehen.

Allerdings ist dieser Nachweis nicht leicht zu führen. Von Beginn an war die europäische Integration ein Elitenprojekt. ${ }^{12}$ Solange die politische Brisanz europäischer Entscheidungen gering war, ähnelten die Integrationseinstellungen der Bürgerinnen und Bürger den Einstellungen gegenüber anderen internationalen Regimen. Die europäische Integration wurde wohlwollend, aber mit geringer Aufmerksamkeit begleitet. Hierfür haben Lindberg und Scheingold ${ }^{13}$ den Begriff des "permissiven Konsenses " geprägt - und vermuteten, dieser Konsens werde an Grenzen stoßen, sobald der Integrationsprozess kritische Bereiche nationaler Souveränität berühre. ${ }^{14}$ Diese Vermutung erscheint heute bemerkenswert weitsichtig. Seit der Schaffung des gemeinsamen Binnenmarkts mehrten sich die Anzeichen für eine wachsende Kluft zwischen den Integrationspräferenzen der Eliten einerseits und dem Rest der Bevölkerungen andererseits und wurden durch eine Serie verlorener nationaler Referenden zu wichtigen Integrationsschritten zur Gewissheit. Europäische Entscheidungen werden zunehmend als politische Entscheidungen wahrgenommen, und zwar unter dem Vorzeichen einer beträchtlichen Skepsis gegenüber dem europäischen Projekt. Der "permissive Konsens « ist einer »negativen Politisierung " gewichen. ${ }^{15}$

Aus Sicht der Kommission wird die Erfolgs-Legitimation der Integrationspolitik also gerade in einer Phase prekär, in der sie angesichts historischer Schritte zur Vertiefung eigentlich besonders dringlich gebraucht würde. Aus dieser Konstellation heraus wird verständlich, warum es im Interesse der Kommission liegt, den Integrationswillen der Bürgerinnen und Bürger ausgeprägter darstellen zu können, als er eigentlich ist.

\section{Das Eurobarometer und die Regeln guter Umfrageforschung}

Wir wollen nachfolgend prüfen, ob sich Anzeichen dafür finden, dass die Kommission ihre Frage- und Interpretationsmacht nutzt, um die Befunde des Eurobarometers in eine erwünschte Richtung zu lenken. Unsere Aufmerksamkeit gilt dabei sowohl der Formulierung und Anordnung von Fragen und Antwortmöglichkeiten als auch der Interpretation von Ergebnissen. Die empirische Grundlage dieser Prüfung sind die 93 deutschsprachigen Fragebögen, die zwischen 1995 und 2010 zum

12 Haller 2009.

13 Lindberg, Scheingold 1970, S. 38.

14 Ebd., S. 274.

15 Hooghe, Marks 2007, 2008; Offe 2003, S. 253. 
Einsatz kamen (in einem Fall diskutieren wir zudem eine Frage aus einem Flash Eurobarometer). Dabei handelt es sich um die Umfragen 43.0 bis 73.4.

Die Suche nach Auffälligkeiten in den Eurobarometer-Fragebögen setzt ein Prüfraster voraus, anhand dessen sich Verletzungen der wissenschaftlichen Redlichkeit identifizieren lassen. Seit George Gallup in den 1930er Jahren die Grundlagen der Stichprobenbefragung entwickelte, haben sich die Kenntnisse über demoskopische Fehlerquellen kumuliert und verdichtet. Hierbei wurde herausgearbeitet, dass Variationen im Aufbau von Fragebögen sowie bei der der Formulierung von Fragen und möglichen Antworten, die dem Laien kaum bewusst sind, Verzerrungseffekte auslösen können, die sich durch Beachtung einiger Regeln guter Umfrageforschung minimieren lassen. ${ }^{16}$ In mehreren Standardwerken ${ }^{17}$ werden diese Regeln $\mathrm{zu}$ »zehn Geboten" guter Umfrageforschung zusammengefasst, auf die wir uns nachfolgend beziehen werden. ${ }^{18}$

Diesen zehn Grundregeln zufolge müssen alle gestellten Fragen ...

1. ... verständlich und wenig komplex sein.

2. ... hypothetische Formulierungen vermeiden.

3. ... auf Informationen abzielen, über die die Befragten tatsächlich verfügen.

4. ... doppelte Stimuli vermeiden.

5. ... unklare Begriffe vermeiden.

6. ... einen eindeutigen zeitlichen Bezug aufweisen.

7. ... mit multiplen Themen in unterschiedliche Richtungen gepolt werden.

8. ... Unterstellungen und suggestive Formulierungen vermeiden.

9. ... vorgegebene Antwortkategorien verwenden, die überschneidungsfrei, ausbalanciert und vollständig sind.

10. ... Wirkungen auf nachfolgende Fragen im Fragebogen-Kontext vermeiden.

Der Rückgriff auf diese zehn Grundregeln hat zwei Vorteile. Erstens, sie sind auch für jene, die selbst nicht regelmäßig mit Demoskopie in Berührung kommen, intuitiv verständlich und nachvollziehbar; wo gleichwohl weitere Erläuterungen zu den Hintergründen einzelner Regeln notwendig erscheinen, erfolgen diese im nachfolgenden Abschnitt. Zweitens, die zehn Regeln gehören anerkanntermaßen zum »kleinen Einmaleins « der Umfrageforschung. Sie sind ohne Zweifel auch den Wissenschaftlerinnen und Wissenschaftlern des Eurobarometers und den verantwortlichen Beamten bekannt. Lassen sich also - über den Einzelfall hinaus - grobe Regelverstöße identifizieren, dann darf angenommen werden, dass diese vorsätzlich erfolgten.

Nachfolgend berichten wir über unsere Funde. Um unsere Angaben nachvollziehbar zu machen, geben wir die entsprechenden Fragen in Kästchen wieder. ${ }^{19}$ Hierbei

16 Kaase 1999, S. 20.

17 Payne 1951; Porst 2008; Diekmann 2005.

18 Die konkreten Formulierungen weichen in den zitierten Standardwerken voneinander ab, die Inhalte der Regeln sind aber im Wesentlichen deckungsgleich.

19 Diskrepanzen in der Schreibweise (zwischen den Fragekästen wechseln der Einsatz von Groß- und Kleinschreibung sowie Satzzeichen am Schluss der Fragen) entstammen den Originalfragen. 
informiert die obere Zeile stets darüber, in welcher Befragung die jeweilige Frage zum ersten und (im Falle mehrfach eingesetzter Fragen) zum letzten Mal gestellt wurde. Es folgen der Wortlaut der Frage und die Antwortmöglichkeiten. Wenn wir im Fließtext auf bestimmte Befragungswellen verweisen, geschieht dies unter Nennung der Nummer des entsprechenden Eurobarometers (Beispiel: EB 69.2). Verweisen wir hingegen auf die von der Kommission vorgenommenen schriftlichen Interpretationen der Befunde, dann sind die entsprechenden Berichte in das Literaturverzeichnis aufgenommen (beispielsweise EB 1997).

\section{Regelverletzungen}

\subsection{Die Gebote der Verständlichkeit, der Vermeidung hypothetischer Fragen und der adäquaten Berücksichtigung vorgefundener Grade an Informiertheit}

Wir handeln die ersten drei Grundregeln in einem gemeinsamen Unterabschnitt ab, weil sie auf eng verwandte Sachverhalte zielen. Wie auch die Grundregeln 4 bis 6 fordern sie, Verzerrungen zu vermeiden, die aus systematischen Überforderungen der Probanden resultieren. Den Regeln 1 bis 3 zufolge sollen Fragen verständlich, möglichst wenig komplex, nicht hypothetisch und der Informiertheit der Befragten angemessen sein. Wenden wir uns zunächst den eng verwandten Regeln 1 und 3 zu und betrachten zu diesem Komplex die in Kasten 1 wiedergegebene Frage, die auch bei von Arnim diskutiert wird. ${ }^{20}$

Kasten 1: Umfrage 60.1 (2003); zuletzt 62.0 (2004)

(Q29) Wie ist Ihre Meinung zu den folgenden Vorschlägen? Bitte sagen Sie mir für jeden Vorschlag, ob Sie dafür oder dagegen sind.

- Eine Europäische Währungsunion mit einer gemeinsamen Währung, nämlich dem Euro.

- Eine gemeinsame Außenpolitik der Mitgliedsstaaten der EU gegenüber anderen Staaten.

- (...5 weitere Themen...)

- Die Tatsache, dass sich die Europäische Kommission aus Kommissionsmitgliedern aus jedem Mitgliedsland zusammensetzt.

(dafür) (dagegen) (weiß nicht)

Der EU-Osterweiterung der Jahre 2005 und 2007 ging eine Debatte über notwendige Anpassungen im Gefüge der europäischen Organe voraus. Von dieser Debatte wurde auch die Struktur der Kommission erfasst. Stellten die großen Mitgliedsstaaten bis dahin jeweils zwei Kommissare und die kleinen nur einen, favorisierte die Kommission ein Reformmodell, dem zufolge nach vollzogener Erweiterung jedes Land nur noch einen Kommissar entsenden sollte. ${ }^{21}$

20 Siehe von Arnim 2006, S. 101.

21 Tatsächlich war das Reformmodell noch wesentlich komplexer: Es sollte nur noch eine Teilgruppe der Staaten stimmberechtigt sein, zudem sollte die stimmberechtigte Teilgruppe nach einem bestimmten Rotationsprinzip wechseln; ausführlich: Wessels 2008, S. 239-244. 
In der in Kasten 1 wiedergegebenen Frage stand die Zusammensetzung der Kommission am Ende einer Liste von acht »Schlüsselthemen «, von denen keines näher erläutert wurde. Zwar gilt für die politische Demoskopie generell, dass abgefragte Haltungen naturgemäß häufig auf komplexe Probleme zielen. Während aber viele Befragte zumindest eine ungefähre Vorstellung von den auf der inhaltlichen Ebene angesiedelten Implikationen einer Währungsunion oder einer gemeinsamen Außenpolitik haben könnten, muss dies für den auf der operationalen Ebene angesiedelten Aufbau der Kommission bezweifelt werden. ${ }^{22}$ Jeden Mitgliedsstaat bei der Besetzung der Kommission zu berücksichtigen klingt einerseits gerecht. Diese Gerechtigkeits-Implikation ist unmittelbar erfassbar. Andererseits warnten Kritiker vor einer Aufblähung der Kommission sowie den Gefahren einer schleichenden Intergouvernementalisierung. Einsichten in diese Implikationen sind nicht unmittelbar erfassbar, sondern erfordern spezifische Informationen. Angesichts dieser Asymmetrie sollte nicht überraschen, dass der Vorschlag in den Umfragen Zustimmungswerte von über 70 Prozent $^{23}$ erzielen konnte, was die Kommission als »ausgeprägten Wunsch nach einem Kommissar pro Mitgliedsstaat « (und folglich als überwältigende Unterstützung für ihr Reformmodell) interpretierte. ${ }^{24}$ Es erscheint aber fraglich, ob von der Berücksichtigung dieser Frage überhaupt ein sinnvolles Resultat zu erwarten war.

Wenden wir uns nun der in Kasten 2 wiedergegebenen Frage zu.

Kasten 2: Flash 211 (2007)

(Q4) Sollte Europa Ihrer Meinung nach ein eigenes Navigationssystem entwickeln oder sollte Europa auf die amerikanischen, russischen oder chinesischen Systeme angewiesen sein?

- die EU sollte ihr unabhängiges System errichten

- $\quad$ es gibt keinen Bedarf für ein unabhängiges System

- weiß nicht

22 Ähnlich kritisiert Schmitt überfordernde Fragen nach policy means anstelle von policy ends; Schmitt 2003, S. 248. Vergleiche zum allgemein hohen Grad an Komplexität vieler im Eurobarometer gestellter Fragen auch Karmasin, Pitters 2008, S. 443-444.

23 Im gesamten Beitrag werden stets die gesamteuropäischen Ergebnisse wiedergegeben. Ist anderes der Fall, wird auf diesem Umstand explizit hingewiesen (siehe Abschnitt 4.5).

24 EB 2004, S. 159. Siehe zur Strategie der Überinterpretation nicht verwunderlicher Befragungsresultate beispielsweise auch EB 71.1 (2009), zuletzt EB 73.4 (2010), QD9. Hier wurden die Probanden unter anderem gefragt, ob sich die Finanzkrise mit folgender Maßnahme wirksam bekämpfen ließe: »Dass die Mitgliedstaaten der EU ihre jeweilige Wirtschafts- und Finanzpolitik stärker aufeinander abstimmen. "Die Befragten konnten zwischen »sehr wirksam «, ziemlich wirksam ", »nicht sehr wirksam « und »unwirksam « wählen. In ihrer Interpretation der Ergebnisse fasste die Kommission die Kategorien "sehr wirksam " und "ziemlich wirksam « zu einer Kategorie zusammen und folgerte in einer Presseerklärung: 75 Prozent der »EU citizens favour stronger European economic governance " - obwohl von einer spezifisch europäischen Regelung in der gestellten Frage keine Rede war; http://europa.eu/rapid/pressReleasesAction.do?reference=IP/10/1071 (Zugriff vom 18.04.2012).

Leviathan, 40. Jg., 3/2012 
(Q5) Haben Sie schon vom europäischen Galileo-Projekt gehört? Ja / Nein

(Q6) Galileo ist der Name des Positionierungssystems, das die EU vor sieben Jahren zu entwickeln begonnen hat. Derzeit sieht es so aus, als ob zusätzliche öffentliche Gelder (ca. 2,4 Milliarden Euro, was den Kosten für ca. 400 km Autobahn entspricht) nötig wären, um das Galileo-System fertigzustellen. Was bevorzugen Sie:

- Die EU sollte die nötigen Mittel sicherstellen, um Galileo so bald wie möglich fertigzustellen

- Die EU sollte keine zusätzlichen Mittel sicherstellen, selbst wenn dies bedeuten würde, dass das Projekt deutlich verzögert wird oder sogar scheitert

- weder noch / keine Angabe

Hier geht es um eine 2007 durchgeführte Telefonumfrage (ein Flash Eurobarometer) zum umstrittenen europäischen Satellitennavigationssystem »Galileo «, für das sich die Kommission einsetzte. Vielen Leserinnen und Lesern wird der suggestive Charakter der Kostenerläuterung in Q6 auffallen. Worauf es uns hier aber ankommt, ist die auf dem Weg von der Frage zur Interpretation erfolgende Verletzung der Grundregel 3. Richtigerweise wird Q6, die direkt auf das »Galileo «-Projekt zielt, die Filterfrage Q5 vorangestellt. Ungefähr 80 Prozent der Probanden beantworteten Q4 mit der ersten Antwortmöglichkeit, und 64 Prozent gaben bei Q6 an, für das Projekt auch öffentliche Finanzmittel bereitstellen zu wollen. Auf Grundlage dieses Resultats betonte die Kommission in ihrer Presseerklärung, dass eine »überwältigende Mehrheit « der Europäer für die Errichtung eines eigenen Navigationssystems sei. ${ }^{25}$ Problematisch ist hier, dass 60 Prozent der Befragten in ihrer Antwort auf Q5 angaben, noch nie von dem »Galileo «-Projekt gehört zu haben. Gleichwohl flossen ihre Antworten in die Einstellungsfragen Q4 und Q6 ein. Die Verletzung der Grundregel 3 erscheint hier besonders evident, weil die Initiatoren der Befragung durch Einbau der Filterfrage Q5 dokumentierten, dass sie sich des Problems mangelnder Informiertheit der Befragten durchaus bewusst waren. ${ }^{26}$

Unserer Ansicht nach findet sich das Problem der (strategischen) Scheinabfrage nicht vorhandenen Wissens auch immer wieder in den Standardfragen des Eurobarometers. Ein Beispiel hierfür ist die häufig gestellte Frage nach dem Vertrauen in die EU-Institutionen. ${ }^{27}$ Möglicherweise darf vorausgesetzt werden, dass die Zusammensetzung und die Kompetenzen von Rat, Parlament und Kommission allgemein bekannt sind (bereits bei letzterem allerdings würden wir erhebliche Zweifel anmelden; vgl. oben). Die Fragen zum Institutionenvertrauen schließen aber auch den Europäischen Rechnungshof, den Wirtschafts- und Sozialausschuss und den Europäischen Ombudsmann mit ein. Auf die Frage, ob man von letzteren drei Institu-

\section{EB 2007.}

26 Am Rande sei erwähnt, dass kurz darauf bekannt wurde, dass die Kommission intern schon seit geraumer Zeit von Gesamtkosten in Höhe von mindestens zehn Milliarden Euro ausging (Financial Times Deutschland vom 6. Juni 2007, S. 8); vergleiche dazu aber die (ohnehin suggestive) Kostenangabe in Q6.

27 Auf diesen Umstand weist auch Haller (2009, S. 359) hin. 
tionen schon einmal gehört oder gelesen habe, antworten regelmäßig große Mehrheiten mit " nein «, sollen aber trotzdem die Frage beantworten, ob sie diesen »eher " oder "eher nicht" vertrauen (zuletzt in EB 73). Warum sollten die Befragten von einem Rechnungshof, über dessen Aufgaben und Tätigkeiten keine Informationen vorliegen, antworten, dass sie ihm misstrauen? Fragen wie diese münden in den Berichten dann in offensichtlich sinnlose Erfolgsmeldungen wie: »[B]esonders hoch ist das Vertrauen in den Bürgerbeauftragten in Finnland « ${ }^{28}$

Die Grundregel 2 verweist auf die problematischen Aspekte hypothetischer Fragen. Sie verlangen von den Befragten, sich eine für sie nicht reale Situation vorzustellen und diese zu bewerten. Solche Fragen sollten nur gestellt werden, wenn sie für möglichst simple Konstrukte stehen. So werden im Eurobarometer beispielsweise regelmäßig die Erwartungen hinsichtlich des eigenen Lebens nach Ablauf eines Jahres abgefragt. Dabei geht es um hypothetische Sachverhalte, die aber so lebensnah sind, dass erwartet werden kann, dass sich die Befragten tatsächlich mit ihnen auseinandersetzen. Ein Beispiel für einen nicht lebensnahen hypothetischen Sachverhalt ist hingegen die Frage nach den wahrscheinlichen, in zehn Jahren eintretenden Wirkungen der wirtschaftspolitischen Programme der europäischen Organe (Kasten 3).

Kasten 3: 73.4 (2010)

(QC2) Bitte sagen Sie mir für jedes der nachfolgend genannten Ziele, die bis zum Jahr 2020 in der Europäischen Union erreicht werden sollen, ob es Ihrer Meinung nach zu ehrgeizig, ungefähr angemessen oder zu bescheiden ist.

- Die Ausgaben für Forschung und Entwicklung sollten jährlich 3\% des in der EU erwirtschafteten Vermögens betragen

- Die Verringerung des Ausstoßes von Treibhausgasen in der EU um mindestens 20\%Punkte bis 2020 gegenüber dem Stand von 1990

- Die Erhöhung des Anteils erneuerbarer Energien in der EU um 20\%-Punkte bis 2020

- Die Erhöhung der Energieeffizienz in der EU um 20\%-Punkte bis 2020

- Der Anteil junger Leute, die die Schule ohne Abschluss verlassen, sollte nicht mehr als $10 \%$ der Schüler betragen

(zu ehrgeizig) (ungefähr angemessen) (zu bescheiden) (weiß nicht / keine Angabe)

Der lange Zeitraum von zehn Jahren und die vielen Details stellen hier keine lebensnahen, sondern abstrakte Rahmenbedingungen dar. Hypothetische Antwortmöglichkeiten wie »bis 2020 zwanzig Prozent weniger Treibhausgase als 1990 « überfordern die Befragten kognitiv, zumal als geforderte Rechenleistung ohne Bezugsgröße. Wer sollte in der Lage sein, bewerten zu können, ob solche Ziele »zu ehrgeizig ", " ungefähr angemessen " oder »zu bescheiden " sind? Die Gefahr erscheint hoch, hier nicht die Einschätzungen der Probanden, sondern sozial erwünschte Antworten einzufangen. In der Tat ergab die Umfrage, dass zwei Drittel der Befragten die Ziele als angemessen bewerteten. Interessant ist nun, dass auch die Eurobarometer-Einheit bei der Interpretation der Ergebnisse anmerkte, dass »dieses Ergebnis möglicherweise eher als Ausdruck einer Erwartung interpretiert 
werden kann ... denn als fundierte Bewertung der einzelnen Ziele «. ${ }^{29}$ Wurde die Frage also nachweislich als nicht sinnvoll erkannt, waren die entsprechenden Skrupel bereits in den Folgeberichten verflogen. Denn im darauffolgenden Report ließ die Kommission verlautbaren: »Die europäischen Bürger bewerten die Zielvorgaben der Kommission als angemessen $«{ }^{30}$

\subsection{Die Verbote doppelter Stimuli sowie unklarer Begriffe und das Gebot eindeutiger zeitlicher Bezüge}

Auch die Grundregeln 4, 5 und 6 zielen auf die Vermeidung der Überforderung der Befragten. Fragen sollen doppelte Stimuli vermeiden, also nicht mehrere Themen gleichzeitig abfragen; es soll eindeutig sein, worauf die in den Fragen gewählten Begriffe zielen; und unklare zeitliche Bezüge wie »in weiter Ferne « oder »in naher Zukunft « sind ebenfalls zu vermeiden. Zwar fanden wir bei unserer Durchsicht der Eurobarometer-Bögen den einen oder anderen Zweifelsfall, nicht aber eklatante Regelverstöße, die mutmaßlich darauf zielten, die Antworten der Befragten in eine erwünschte Richtung zu lenken. In Bezug auf diese drei Regeln erscheinen die Eurobarometer-Befragungen daher weitgehend » redlich".

\subsection{Das Gebot wechselnder Polung multipler Fragen}

Grundregel 7 besagt, dass zu bewertende Aussagen in Fragebatterien mit multiplen Themen in unterschiedliche Richtungen zu polen sind. Hintergrund dieses Gebots ist das Problem der inhaltsunabhängigen Zustimmungstendenz (Akquieszenz), nach dem manche Probanden dazu neigen, stets mit »stimme zu « bzw. »ja « zu antworten. Mit Aussagen, die einseitig positiv oder negativ gepolt sind, lassen sich Befunde daher in erwünschte Richtungen lenken. ${ }^{31} \mathrm{Um}$ Informationen über Grade an Akquieszenz zu erhalten, empfiehlt es sich daher, einer Aussage wie »Das Organ X braucht mehr Kompetenzen « auch ein invers gepoltes Angebot wie »Die Macht des Organs X sollte begrenzt werden « entgegenzustellen.

Fragebatterien, bei denen Probanden Aussagen mit »stimme zu « oder »lehne ab « bewerten sollen, finden sich im Eurobarometer häufig. Bei näherer Betrachtung fällt jedoch auf, dass ein Teil der vorgegebenen Aussagen zu EU-Themen eben nicht ausgeglichen, sondern einseitig positiv gepolt wird. Betrachten wir das Beispiel in Kasten 4 .

29 EB 2010 b, S. 208.

30 EB 2011, S. 19.

31 Diekmann 2005, S. 413. 
Kasten 4: 69.2 (2008)

(QA15 a) Sagen Sie mir bitte für jede der folgenden Aussagen, ob Sie ihr eher zustimmen oder sie eher ablehnen.

- Ich fühle mich sicherer, weil Deutschland ein Mitglied der EU ist.

- Ich habe das Gefühl, dass wir wirtschaftlich stabiler sind, weil Deutschland zur Eurozone gehört.

- Meine Stimme zählt in der Europäischen Union.

- Meine Stimme zählt in Deutschland

- Meine Meinung zu europäischen Themen wird von der Bundesregierung berücksichtigt.

- Meine Meinung zu europäischen Themen wird von den Mitgliedern des EP berücksichtigt.

- Meine Meinung zu europäischen Themen wird von der Kommission berücksichtigt

- Ich verstehe, wie die EU funktioniert.

- Die Stimme Deutschlands zählt in der EU

- Die Interessen Deutschlands werden in der EU gut berücksichtigt.

- Die Europäische Union zwingt Deutschland ihre Sichtweise auf.

(stimme eher zu) (lehne eher ab) (weiß nicht / keine Angabe)

Diese Fragebatterie kommt seit der Eurobarometer-Umfrage vom Herbst 2001 regelmäßig zum Einsatz, teils um neue Aussagen ergänzt, teils um alte gekürzt. In den Aussagen 1, 3, 4, 8, 9 und 10 wird der jeweilige Ländername eingesetzt (in unserem Beispiel: Deutschland). Bemerkenswert ist hier, dass nahezu alle Aussagen »europafreundlich « gepolt sind - und das, obwohl Sätze wie »Die Stimme Deutschlands zählt in der EU « und »Die Interessen Deutschlands werden in der EU gut berücksichtigt « logisch nahe beieinander liegen. Gerade in so einem Fall wäre der Rückgriff auf eine inverse Polung angezeigt gewesen. Als nun im Frühjahr 2008 mit dem Satz »Die EU zwingt Deutschland ihre Sichtweise auf « erstmals auch eine negative Aussage bereitgestellt wurde (siehe Item 10) und diese umgehend die höchsten Zustimmungswerte erzielte, nahm man sie zwei Umfragen später wieder aus dem Programm. Am Rande sei erwähnt: Ebenso erging es der zeitgleich aufgenommenen Aussage zur Berücksichtigung von Bürgermeinungen durch die Kommission, der trotz positiver Polung lediglich 25 Prozent zustimmten. ${ }^{32}$ Hier ist offensichtlich, dass die Kommission auf »integrationistische " Ergebnisse abzielt, »euroskeptische « Ergebnisse hingegen unterbindet.

Einen weiteren Hinweis auf intendierte Ergebnislenkung fanden wir bei dem Themenkomplex »EU-Erweiterung «. Seit 1997 werden die Befragten regelmäßig dazu aufgefordert, verschiedenen Aussagen nach dem Schema »Je mehr Erweiterung, desto ..." zuzustimmen oder diese abzulehnen. Bereits seit Umfrage 48.0 (Q37) geschieht dies unter redlicher Berücksichtigung des Gebots wechselnder Polung. Es

32 Das Item »Die EU zwingt Deutschland ihre Sichtweise auf « wurde in den Umfragewellen 69.2 und 70.1 bereitgestellt und erreichte mit 60 Prozent umgehend die höchsten Zustimmungswerte aller Aussagen. Es wurde beim nächsten Einsatz der Fragebatterie in Umfragewelle 71.3 (QA12 a) jedoch nicht mehr erhoben. Die Items » Meine Meinung zu europäischen Themen wird von der Kommission «bzw. »von den Mitgliedern des EP berücksichtigt « erreichten lediglich 25 bzw. 22 Prozent Zustimmung und wurden seither nicht wieder abgefragt. 
müssen also auch negativ gepolte Aussagen wie »... desto mehr Arbeitslosigkeit « bewertet werden. Diese wissenschaftlich korrekte Vorgehensweise fand jedoch ausgerechnet dann eine Unterbrechung, als die Kommission im Jahr 2001 eine Spezialumfrage zur EU-Osterweiterung durchführte. Plötzlich waren die positiv gepolten Aussagen in klarer Überzahl, und zuvor negativ gepolte Aussagen tauchten in inverser Formulierung auf, so dass nun Aussagen wie »Die Erweiterung wird mehr Arbeitsplätze schaffen " bewertet werden mussten. ${ }^{33}$ Obwohl die Ergebnisse aus Sicht der Kommission auch hier gewiss nicht befriedigend ausfielen, konnte sie Negativmeldungen wie » 50 Prozent der Deutschen erwarten mehr Arbeitslosigkeit « nunmehr vermeiden. Stattdessen hieß es einseitig positiv: »20 Prozent erwarten mehr Jobs « ${ }^{34}$ Die Tatsache, dass dieser Regelverstoß in der Spezialumfrage zur Osterweiterung offenbar gezielt eingefügt wurde - er tauchte weder davor noch danach (wieder) auf -, bestärkt uns in der Interpretation, dass das Eurobarometer Fragekonstruktionen gezielt nutzt, um Antworten auf politisch besonders brisante Fragestellungen in eine erwünschte Richtung zu lenken.

\subsection{Das Verbot von Unterstellungen und Suggestivfragen}

Unterstellungen und Suggestivfragen sind geeignet, Antwortreaktionen in eine vom Fragesteller erwünschte Richtung zu lenken. ${ }^{35}$ Solche Formulierungen suggerieren dem Befragten, bei welcher Antwort es sich um die vom Interviewer und/oder von der Bezugsgruppe des Befragten erwünschte Alternative handelt, der zu widersprechen den Interviewer enttäuschen oder eine Abweichung von Gruppennormen bedeuten würde. Auch das Verbot von Unterstellungen und Suggestivfragen zielt also auf die Vermeidung von Verzerrungen der Befunde durch Effekte sozialer Erwünschtheit. ${ }^{36}$ Betrachten wir hierzu die Frage in Kasten 5.

Kasten 5: 44.0 und 44.1 (1995)

(Q59) Manche sagen, dass die Länder Mittel- und Osteuropas, wie z.B. die Tschechische Republik, Ungarn, Polen oder die Slowakei, Mitglied der Europäischen Union werden sollen. Wie ist ihre Meinung dazu? Sollen sie...

- ...in weniger als fünf Jahren Mitglied werden,

- $\quad$...in den nächsten fünf bis zehn Jahren oder

- ...in mehr als zehn Jahren?

- Ich finde nicht, dass diese Länder Mitglied der EU werden sollten (INT.: Nur falls spontan genannt!)

- weiß nicht

33 In Umfrage 55.0 (Q22) standen acht positiv gepolten Aussagen lediglich zwei negativ gepolte gegenüber.

34 EB 2001, S. 3.

35 Porst 2008, S. 103.

36 Faulbaum et al. 2009, S. 172. 
Der Einleitungssatz zu dieser Frage erfüllt das Kriterium der Suggestion, weil er den aufgerufenen Sachverhalt einseitig positiv konnotiert, ließe sich doch mit demselben Recht auch als Einleitungssatz formulieren, dass manche vor einer unkontrollierten Ausdehnung der EU warnen. ${ }^{37}$ Nicht zum ersten Mal begegnen wir hier dem Umstand, dass bei Fragen, in denen bestimmte Antworten offensichtlich besonders erwünscht waren, mehrere Grundregeln gleichzeitig verletzt werden. Denn die Probanden werden dahingehend vor vollendete Tatsachen gestellt, dass ihnen lediglich drei unterschiedliche Zeiträume für die Osterweiterung zur Auswahl gestellt werden, während die Möglichkeit einer grundsätzlichen Ablehnung nur dann berücksichtigt wurde, wenn die Befragten den Interviewer spontan darauf aufmerksam machten. Dies geschah in beachtlichen 50 Prozent der Fälle; ${ }^{38}$ wir können nur mutmaßen, wie das Ergebnis gelautet hätte, wäre den Interviewten eine redlich formulierte Frage vorgelegt worden.

Wenden wir uns einem anders gelagerten Beispiel für den Einsatz suggestiver Formulierungen im Eurobarometer zu (Kasten 6).

Kasten 6: 59.1 (2003)

(Q21) Ich lese Ihnen nun einige Maßnahmen vor, die die Europäische Union ergreifen könnte. Sagen sie mir bitte für jede dieser Maßnahmen, ob sie ihrer Meinung nach vorrangig behandelt werden sollte oder nicht.

- Erfolgreiche Erweiterung der Europäischen Union um neue Mitgliedsländer aufzunehmen

- Mehr Bürgernähe in Europa erreichen, z.B. indem man die Bürger besser über die Europäische Union, ihre Politik und ihre Institutionen informiert

- Die einheitliche Europäische Währung, den Euro, erfolgreich einführen

- (...12 weitere Fragen)

(vorrangig behandeln) (nicht vorrangig behandeln) (weiß nicht)

Die Leserinnen und Leser werden schnell erkennen, dass zwei der hier aufgeführten Antwortmöglichkeiten suggestive Formulierungen enthalten, denn was sollte dagegen sprechen, »erfolgreiche « Maßnahmen vorrangig durchzuführen? Interessant ist an dieser Regelverletzung nun, dass die Frageformulierung im Falle der Osterweiterung offenbar als suggestiv erkannt wurde. Sie wurde in einer nachfolgenden Befragung (EB 60) durch eine neutrale Formulierung ersetzt (»neue Mitgliedsstaaten aufnehmen «). Wie nicht anders zu erwarten, führte der Verzicht auf die Suggestion zu einem veränderten Ergebnis. Statt 33 Prozent (in EB 59) sprachen sich nun nur noch 27 Prozent (in EB 60) dafür aus, dass die Erweiterung vorrangig behandelt werden sollte. Die nahezu konstante Beantwortung der ebenfalls in beiden Erhebungen gestellten Frage, ob man für oder gegen eine Erweiterung sei (46 Prozent dafür in EB 59, 47 Prozent dafür in EB 60) deutet darauf hin, dass die Diskrepanz von sechs Prozentpunkten in der Tat nicht einem Meinungsumschwung in Erwei-

37 Siehe etwa auch Q47 in EB 47.0: »Einige sagen, dass der Euro die EU in die Lage versetzen wird, mächtiger und wettbewerbsfähiger zu sein."

38 EB 1995.

Leviathan, 40. Jg., 3/2012 
terungsfragen, sondern dem Verzicht auf die manipulative Fragestellung geschuldet war.

In ihrer Interpretation verzichtete die Kommission aber darauf, auf diesen Umstand hinzuweisen, ${ }^{39}$ und betonte lediglich, dass die Befürwortung der Unionsbürger für das Prinzip der Erweiterung ... praktisch unverändert « geblieben war: »[E]ine relative Mehrheit ist dafür « (EB 2003, S. 76). Besonders eklatant erscheinen die Verstöße gegen die Regeln guter Umfrageforschung hier, weil das »natürliche Experiment « der Stellung derselben Frage mit und ohne Suggestion in zwei aufeinanderfolgenden Befragungen den Effekt der gezielten Manipulation (»erfolgreich einführen «) plastisch vor Augen führte - die Suggestion in der »Euro-Frage « (»den Euro erfolgreich einführen «) aber gleichwohl ab der Umfrage 59.1 unverändert blieb und anschließend noch in 21 weiteren Befragungen zum Einsatz kam. Wir dürfen ausschließen, dass die verzerrende Wirkung des Wortes »erfolgreich «im Falle der Erweiterungsfrage erkannt wurde, im Falle der zwei Zeilen darunter gestellten Euro-Frage aber ohne Vorsatz unerkannt blieb. ${ }^{40}$

\subsection{Das Gebot ausbalancierter Antwortkategorien}

Die neunte Grundregel guter Umfrageforschung fordert, dass bei (teil)geschlossenen Fragen alle vorgegebenen Antwortkategorien vollständig, überschneidungsfrei und positiv wie negativ ausbalanciert sein müssen. ${ }^{41}$ Verletzungen dieses Gebots können die Ergebnisse - gewollt oder ungewollt - verzerren.

Wenden wir uns zunächst einem speziellen Problem zu: dem Fehlen mittlerer Antwortkategorien. Der Verzicht auf eine indifferente Kategorie allein deutet nicht auf manipulative Absichten hin. In Kombination mit manipulativen Elementen ist das Fehlen einer Mittelkategorie aber geeignet, Verzerrungseffekte zu verstärken. Betrachten wir hierzu die Frage in Kasten 7, bei der es sich um eine der wichtigsten Standardfragen des Eurobarometers handelt. ${ }^{42}$ Die Befragten sollen angeben, wie sie die Effekte der EU-Mitgliedschaft auf ihr Land einschätzen.

Kasten 7: zuletzt $73.4(2010)$

(QA10 a) Hat Ihrer Meinung nach Deutschland insgesamt gesehen durch die Mitgliedschaft in der Europäischen Union Vorteile, oder ist das nicht der Fall?

- Vorteile

- ist nicht der Fall

- weiß nicht

39 Das ist in diesem Fall bemerkenswert, weil in den Berichten auf Änderungen von Fragestellungen in der Regel hingewiesen wird.

40 Die Frage nach »vorrangigen « bzw. » nicht vorrangigen « Maßnahmen war von Umfrage 59.1 (2003) bis 66.1 (2006) in ausnahmslos jedem Standard- und Spezial-Eurobarometer enthalten und blieb - abgesehen von der angesprochenen "Erweiterungsfrage « - dabei unverändert. Seither wird die Frage in dieser Form nicht mehr gestellt.

41 Faulbaum et al. 2009, S. 188.

42 Dieses Problem wird auch diskutiert bei von Arnim 2006, S. 100. 
Hier tritt eine unsaubere Fragestellung - die Möglichkeit von »Vorteilen « wird explizit aufgerufen, das logische Gegenstück »Nachteile « hingegen nicht - in Kombination mit einer fehlenden Mittelkategorie (etwa: »beides « oder »weder-noch «) auf. Wer gegenüber dem Interviewer nicht als uninformiert erscheinen möchte, ist also gehalten, sich für eine der Kategorien zu entscheiden. Im Eurobarometer 66 des Jahres 2006 antworteten 49 Prozent der deutschen ${ }^{43}$ Befragten mit »Vorteile «, 41 Prozent mit »keine Vorteile «, und 10 Prozent machten keine Angabe.

Bemerkenswert ist nunmehr, wie sich die Ergebnisse verändern, wenn die Frage sowohl wissenschaftlich korrekt als auch mit einer mittleren Antwortkategorie versehen gestellt wird. Eben dies tat die Forschungsgruppe Wahlen im selben Jahr $2006 .{ }^{44}$ Der Wortlaut der Frage lautete: »Die Mitgliedschaft in der EU bringt alles in allem gesehen der deutschen Bevölkerung eher ..." u, und die Antwortkategorien waren »Vorteile «, »Nachteile «, »Vor- und Nachteile « sowie » weiß nicht «. Es stellte sich heraus, dass sich 46 Prozent der Befragten der mittleren Kategorie zuordneten. 22 Prozent sahen eher Vorteile, 29 Prozent eher Nachteile, und 3 Prozent machten keine Angabe. Durch scheinbar geringe Umformulierungen hatte sich das Gesamtbild also erheblich verändert. Wie auch immer das Ergebnis der Forschungsgruppe Wahlen zu interpretieren sein mag - die Interpretation muss nunmehr eine substanziell andere sein als jene der Kommission: »Nach wie vor glaubt die Mehrheit, dass ihr Land durch die EU-Mitgliedschaft Vorteile hat « ${ }^{45}$

Kommen wir nun zu dem Problem unbalancierter Antwortkategorien. Ein Beispiel unter vielen findet sich in Kasten 8. Die darin wiedergegebene Frage zielt auf den Nutzen der (damals im Aufbau befindlichen) Währungsunion (EWU), wiederum aus Sicht des jeweiligen Heimatlands der Befragten.

Kasten 8: 50.1 (1998)

(Q58) Welche der folgenden Aussagen kommt Ihrer eigenen Meinung am nächsten?

- Für Deutschland ist die europäische Währungsunion nützlich und bringt Vorteile

- Für Deutschland ist die europäische Währungsunion notwendig, aber sie bringt keine Vorteile

- Für Deutschland bringt die europäische Währungsunion mehr Probleme als Vorteile

- weiß nicht

Auf den ersten Blick mögen diese Fragestellung und die Antwortkategorien fair erscheinen. Bei näherer Hinsicht zeigt sich, dass sie es nicht sind. Denn zwei der drei Antwortmöglichkeiten sind dazu geeignet, die Schaffung der EWU mit Zustimmung zu unterfüttern, während lediglich eine von ihnen keine Zustimmung impliziert. Anzahl und Anordnung der Kategorien scheinen eine indifferente Mittelkategorie

43 Hier referieren wir das auf Deutschland bezogene Befragungsergebnis, um einen sinnvollen Vergleich mit der im nächsten Absatz angesprochenen Umfrage der Forschungsgruppe Wahlen zu ermöglichen.

44 Siehe auch von Arnim 2006, S. 100.

45 EB 2006 a, S. 10.

Leviathan, 40. Jg., 3/2012 
zu suggerieren, die in Wahrheit nicht existiert. Denn wer sich der mittleren Kategorie zuordnet, hat nicht Indifferenz, sondern Zustimmung zur EWU (»ist ... notwendig «) signalisiert. Besondere Brisanz gewinnt dieses Problem, wenn - was zum Standardrepertoire der Kommission gehört - die zustimmenden Kategorien im Zuge der Interpretation zu einer gemeinsamen Kategorie zusammengefasst werden.

In Kasten 9 findet sich eine weitere Spielart unbalancierter Antwortkategorien. Die Probanden sollen aus einer langen Liste an Feststellungen jene auswählen, die am besten beschreiben, was ihnen die EU bedeutet.

Kasten 9: 47.2 (1997); in dieser Form zuletzt 55.1 (2001)

(Q9) Welche der folgenden Aussagen beschreibt am besten, was die EU für Sie persönlich bedeutet? (Liste vorlegen, Mehrfachnennungen möglich)

- $\quad$ ein Weg, für junge Leute eine bessere Zukunft zu schaffen

- $\quad$ eine europäische Regierung

- die Möglichkeit, in Europa überall hinzugehen, wohin ich möchte

- $\quad$ eine Garantie für einen dauerhaften Frieden in Europa

- ein Mittel zur Verbesserung der wirtschaftlichen Situation in Europa

- $\quad$ ein Weg, Arbeitsplätze zu schaffen

- $\quad$ ein Weg, die Rechte der Bürger zu schützen

- viel Bürokratie, eine Verschwendung von Zeit und Geld

- nur ein Traum, eine Utopie

- das Risiko, unsere kulturelle Vielfalt zu verlieren

- $\quad$ sonstiges (INT.: NUR falls spontan genannt!)

- weiß nicht

Auffällig ist hier der Überschuss an positiven Antwortkategorien. Sechs der vorgelegten Antwortmöglichkeiten sind positiv konnotiert, ohne dass ihnen ein logisches Gegenstück gegenüberstehen würde, lediglich zwei von ihnen negativ (vergleiche die von uns eingefügten Vorzeichen am rechten Rand des Kastens). Anderslautende Meinungen werden hier nur dann registriert, wenn sie von den Probanden spontan geäußert werden. Die Wirkung dieses Umstands wird hier zudem durch einen Reihenfolgeneffekt verstärkt, denn alle EU-freundlich gepolten Antwortmöglichkeiten finden sich im oberen Teil der Liste und die beiden EU-kritischen Formulierungen ganz unten (die Demoskopie spricht hier vom zu vermeidenden " primacy effect «). Angesichts der bizarren Konstruktion der Frage lassen sich die durch sie gewonnenen Ergebnisse kaum sinnvoll interpretieren. Die Leserinnen und Leser wird nicht verwundern, dass die Kommission gleichwohl festhielt: »Der allgemeine Eindruck, der sich aus dieser Befragung ergab, ist positiv «. ${ }^{46}$

\subsection{Das Gebot der Vermeidung von Kontexteffekten}

Auch Fragebogenkontexte können Befragungsergebnisse verzerren. Dieser Effekt tritt ein, wenn einmal gestellte Fragen auf spätere Fragen - insbesondere die direkt

46 EB 1997, S. 56. Mittlerweile wurde die Frage umformuliert. Seit EB 57.1 gibt es keinen Überschuss an positiven Antwortmöglichkeiten mehr, und seit EB 65.2 erfolgt eine Anweisung an den Interviewer: »Top to Bottom/Bottom to Top rotieren«. 
im Anschluss gestellten - "ausstrahlen «. Dieser vielfach nachgewiesene Umstand wird in der Demoskopie als Kontext-, Halo-, Positions- oder Reihenfolgeneffekt bezeichnet. ${ }^{47} \mathrm{Um}$ Kontexteffekte so weit wie möglich zu minimieren, empfiehlt es sich daher, durch Probe-Interviews eine möglichst neutrale Reihenfolge der Fragen sicherzustellen.

Betrachten wir hierzu die in Kasten 10 wiedergegebenen drei Fragen aus der im Jahr 2006 durchgeführten "Zukunftsumfrage« der Kommission.

Kasten 10: 65.1 (2006)

(QA12) Was auf dieser Liste halten Sie für den größten Erfolg der Europäischen Einigung? Und was für den zweitgrößten Erfolg?

- Frieden zwischen den Mitgliedsstaaten der EU

- Den freien Verkehr von Personen, Gütern und Dienstleistungen innerhalb der EU

- Den Euro

- Studenten-Austauschprogramme wie ERASMUS

- Die gemeinsame Agrarpolitik

- Spontan: nichts davon

- Spontan: Sonstiges

- Weiß nicht / Keine Angabe

(QA13) Wie würden Sie auf einer Skala von 1 bis 10 die Leistung der Europäischen Union auf jedem der folgenden Gebiete bewerten? Eine "1" bedeutet, dass Sie mit der Leistung der Europäischen Union auf dem jeweiligen Gebiet "überhaupt nicht zufrieden" sind, und eine "10" bedeutet, dass Sie mit der Leistung "sehr zufrieden" sind. Wie ist das mit...

- Dem Kampf gegen die Arbeitslosigkeit

- Dem Schutz sozialer Rechte

- Dem Schutz der Menschrechte

- (...7 weitere Fragen...)

- Die Förderung von Demokratie und Frieden auf der Welt

- Der Zusammenarbeit auf dem Gebiet der Forschung und Innovation

- Der Sicherung einer beständigen Energieversorgung

(QA14) Würden Sie sagen, dass sich die Dinge in Deutschland derzeit ganz allgemein in die richtige Richtung oder in die falsche Richtung entwickeln? Und in der Europäischen Union?

- Dinge entwickeln sich in die richtige Richtung

- Dinge entwickeln sich in die falsche Richtung

- Spontan: Weder noch

- Weiß nicht / Keine Angabe

Bei QA12 handelt es sich um eine neu eingeführte Frage, die unmittelbar vor zwei Einstellungsfragen zu den Leistungen der EU positioniert wurde. QA12 sieht vor, den Befragten eine Liste mit Erfolgen der EU vorzulesen, unter denen diese die zwei

47 Berühmt wurde folgendes Beispielexperiment: Amerikanische Studenten wurden sowohl zur Zufriedenheit mit ihren Datings als auch zu ihrer allgemeinen Lebenszufriedenheit befragt. Die Ergebnisse beider Fragen erwiesen sich als nur sehr schwach positiv korreliert. Positionierte man die Frage nach den Rendezvous aber direkt vor die Frage nach der allgemeinen Lebenszufriedenheit, gewann die Korrelation an Stärke; siehe Strack et al. 1988, S. 435. Die Aktivierung der zuvor aufgerufenen Information »strahlte « also auf die Beantwortung der Folgefrage aus. 
größten benennen sollen. Wir wollen an dieser Stelle von der Konstruktion von QA12 absehen, die die entsprechenden Erfolge der EU unterstellt und der Möglichkeit, dass Befragte diese Unterstellung nicht teilen, keinen Raum gibt. ${ }^{48}$ Unsere Aufmerksamkeit gilt vielmehr einer Verletzung der zehnten Grundregel: QA12 aktiviert gezielt positive Informationen zur europäischen Politik. Daher muss der Möglichkeit Rechnung getragen werden, dass ein Kontexteffekt auf QA13 und QA14 eintritt. Diese potenzielle Fehlerquelle hätte eigentlich auffallen müssen. Während die Fragen zu den Leistungen der EU mittlerweile zum Standardrepertoire des Eurobarometers gehören, ist die »Erfolgsfrage " QA12 seither nicht wieder in den Bögen aufgetaucht. Es muss daher die Frage gestellt werden, ob QA12 in der "Zukunftsumfrage " gezielt vor QA13 und QA14 positioniert wurde, um einen erwünschten Kontexteffekt zu erzielen. ${ }^{49}$ Auch die Interpretation der Kommission nährt den Verdacht, dass bestimmte Ergebnisse in diesem Fall erwünschter waren als andere. Denn obwohl in der Beantwortung von QA14 lediglich 39 Prozent der Probanden angaben, dass sich die EU in die "richtige Richtung " entwickele, ${ }^{50}$ schlussfolgerte die Kommission, dass die "Mehrheit der Befragten ... der Meinung [ist], dass sich die Dinge in der Europäischen Union in die richtige Richtung entwickeln «. ${ }^{51}$

\section{Fazit}

In ihrer Eröffnungsrede zur Eurobarometer-Konferenz »Understanding Public Opinion « im Jahre 2006 merkte die damalige Vizepräsidentin der Kommission Margot Wallström an, dass bereits Gallup der Ansicht war, Demoskopie könne sich belebend auf den demokratischen Prozess auswirken. ${ }^{52}$ Das ist korrekt. Allerdings

48 Die Möglichkeit, dass Befragte keines der Items für den »größten« oder »zweitgrößten « Erfolg der EU halten, ist nur (aber immerhin) als spontane Äußerung der Befragten vorgesehen. Dass Befragte einzelne Items als Misserfolge bewerten, ist hingegen nicht vorgesehen.

49 Tatsächlich veränderten sich die Ergebnisse deutlich, als die »Erfolgsfrage « (in Kasten 10: QA12) in der nachfolgenden Befragung nicht mehr kurz vor QA14 platziert wurde. Hatten in EB 65.1 noch 39 Prozent der Probanden der Aussage zugestimmt, die Dinge in der EU entwickelten sich in die »richtige Richtung ", waren es in EB 66.1 nur noch 33 Prozent. Die Anzahl derer, die mit »falsche Richtung « antworteten, stieg spiegelbildlich um ebenfalls genau sechs Prozentpunkte (von 27 auf 33 Prozent). Allerdings ist unklar, ob diese Veränderungen allein dem entfallenen Kontexteffekt geschuldet sind, denn zwischen EB 65.1 zu EB 66.1 war eine allgemeine Verschiebung in Richtung europessimistischerer Einstellungen zu verzeichnen (vgl. etwa die Befragungsergebnisse zur EU-Mitgliedschaft und zum Vertrauen in die europäischen Institutionen). Diese Verschiebungen waren aber kleiner als sechs Prozentpunkte, so dass wir davon ausgehen, dass zumindest ein Teil der Diskrepanz dem Kontexteffekt geschuldet ist.

5027 Prozent antworteten mit »falsche Richtung «, 23 Prozent mit »weder noch « und 11 Prozent mit » weiß nicht «.

51 EB 2006 b, S. 17.

52 Wallström 2006. 
warnte Gallup gleichzeitig vor staatlich betriebener Meinungsforschung. ${ }^{53}$ Ist der staatliche Auftraggeber gleichzeitig das Objekt der Forschung, entstehen Spielräume für manipulative Lenkung. Eine Identität von Objekt und Subjekt der Umfrageforschung - und damit eine Gelegenheit zur Indienstnahme der Demoskopie für die Verfolgung politischer Ziele - liegt im Fall des Eurobarometers vor. Ausgehend von dem Problem der defizitären demokratischen Legitimation der europäischen Politik, haben wir daher die Frage diskutiert, ob Anzeichen dafür bestehen, dass die Kommission ihre Frage- und Interpretationsmacht dazu benutzt, um den Integrationswillen der Bürgerinnen und Bürger ausgeprägter darzustellen, als er wirklich ist. Dabei haben wir uns auf die Phase seit 1995 konzentriert, in der die Kommission die Verstärkung eines offenen Dialogs mit den Bürgerinnen und Bürgern anstrebte. ${ }^{54}$

In der Tat fanden sich bei unserer Durchsicht der Eurobarometer 43.0 bis 73.4 methodische Auffälligkeiten. So wurden Haltungen zu Sachverhalten abgefragt, die die Befragten aufgrund hoher Komplexität, hypothetischen Gehalts der Fragen oder fehlender Informationen kaum wirklich beurteilen konnten. Es zeigte sich, dass einige Fragebatterien nicht ausgeglichen, sondern einseitig positiv gepolt wurden, und dass das Antwortverhalten durch Unterstellungen und Suggestivfragen in bestimmte Richtungen gelenkt wurde. Des Weiteren fanden wir Belege für den Einsatz nicht ausbalancierter Antwortkategorien und konnten auch den strategischen Einsatz von Kontexteffekten nicht ausschließen. Insgesamt fanden wir Beispiele für die Verletzung von sieben der »zehn Gebote" guter Umfrageforschung. Besonders auffällig waren zudem erhebliche Abstriche bei der redlichen Interpretation integrationspolitisch wichtiger Befunde.

Wohlgemerkt: Es geht uns hier nicht darum, einem methodologischen Purismus das Wort zu reden und an Fehlern zu mäkeln, die auch in der besten Forschung geschehen können. Ohnehin fehlt ein geeigneter Schwellenwert an Abweichungen, oberhalb dessen sich Fragebögen als "unredlich " klassifizieren ließen. Angesichts der Menge der von uns durchgesehenen Fragebögen, so ließe sich argumentieren, ist es nicht verwunderlich, dass sich eine Anzahl an Fehlern fand, die - auf wenigen Seiten komprimiert dargestellt - den Eindruck mangelnder wissenschaftlicher Sorgfalt erwecken. Aus unserer Sicht beschreiben die von uns als Maßstab verwendeten zehn Grundregeln ein Ideal, dem sich gute Umfrageforschung annähern sollte, ohne es wohl jemals vollständig erreichen zu können. Nicht um mangelnde Sorgfalt geht es uns also, sondern um den begründeten Verdacht strategischer Manipulation. Dieser Verdacht ergibt sich aus der systematischen Gerichtetheit der Verstöße gegen die Regeln guter Umfrageforschung. Denn in ausnahmslos allen Fällen waren die Verstöße so gerichtet, dass sie geeignet waren, die Ergebnisse in eine pro-europäische, integrationsfreundliche Richtung zu lenken. Dass dies ohne Intention geschah, wollen wir ausschließen. ${ }^{55}$ Die wissenschaftliche Integrität der Eurobaro-

53 Gallup 1939, S. 10-11; ebenso Pausch 2008, S. 358.

54 Brüggemann 2008, S. 125.

55 Schon die Wahrscheinlichkeit, dass acht Verstöße "zufällig « dieselbe Polung aufweisen, liegt deutlich unter ein Prozent. 
meter-Befragungen muss also mit einem großen Fragezeichen versehen werden. Durch den Einsatz strategischer Manipulation täuscht die Kommission nicht nur die zahlreichen Rezipienten der Eurobarometer-Befragungen über den vermeintlichen Integrationswillen der Bürgerinnen und Bürger, sondern potenziell auch sich selbst.

Von wenigen Ausnahmen abgesehen, ${ }^{56}$ werden die Abstriche an der wissenschaftlichen Seriosität des Eurobarometers von der empirischen Europaforschung bisher im Wesentlichen stillschweigend hingenommen. ${ }^{57}$ Warum dies geschieht, ist unklar. Zwei Möglichkeiten stehen im Raum, und beide wollen wir hier nicht behaupten. Erstens wäre denkbar, dass die Verstöße gegen die Regeln guter Demoskopie in der Integrationsforschung, insbesondere aber von den Nutzern der EurobarometerDaten unerkannt bleiben. Angesichts der in diesem Beitrag aufgezeigten Deutlichkeit der Regelverletzungen erscheint uns dies aber nicht wahrscheinlich. So käme noch eine zweite Möglichkeit in Frage: Man teilt den normativen Integrationismus der Initiatoren des Eurobarometers und drückt deshalb beide Augen zu. Hiervor kann nur nachdrücklich gewarnt werden. Denn dies würde neben der Seriosität des Eurobarometers auch die wissenschaftliche Integrität der Europaforschung infrage stellen. Wie auch immer der wachsenden Kluft zwischen den Integrationspräferenzen der Eliten einerseits und der europäischen Bürgerinnen und Bürger andererseits zu begegnen sein mag: Die in diesem Beitrag aufgezeigte Strategie der Scheinlegitimation darf nicht toleriert werden.

\section{Literatur}

Aldrin, Philippe 2010. »From instruments to the instrumentalisation of `European opinion $<$ a historical sociology of the measurement of opinions and the management of the public space", in A political sociology of the European Union: reassessing constructivism, hrsg. v. Rowell, Jay; Mangenot, Michel, S. 206-224. Manchester: Manchester University Press.

Alter, Karen J. 2001: Establishing the supremacy of European law. Oxford, New York: Oxford University Press.

Alter, Karen J. 2009. The European Court's political power. Selected essays. Oxford, New York: Oxford University Press.

Arnim, Hans Herbert von 2006. Das Europa-Komplott: Wie EU-Funktionäre unsere Demokratie verscherbeln. München: Carl Hanser.

Brüggemann, Michael 2008. Europäische Öffentlichkeit durch Öffentlichkeitsarbeit? Die Informationspolitik der Europäischen Kommission. Wiesbaden: VS Verlag für Sozialwissenschaften.

56 Siehe Aldrin 2010, S. 218; von Arnim 2006, S. 98-102; Haller 2009, S. 358-361; Pausch 2008, 2009; vgl. als Pionierin der Eurobarometer-Kritik Noelle-Neumann 1993, S. 26-34. Anzumerken ist allerdings, dass Aldrin seine Kritik sogleich relativiert: »Without excessively pushing the orientation of the questions and the interpretation of the results, the tool induces an apparent desire for Europe of the pollees by questioning them on their agreement with positively connected attitudes towards dialogue and cooperation between member states «; Aldrin 2010, S. 218, Hervorhebung M.H./B.J.

57 Methodische Kritik am Eurobarometer üben auch Karmasin und Pitters 2008; hier allerdings geht es nicht um den Vorwurf strategischer Ergebnislenkung, sondern um Probleme des Samples und heterogener kultureller biases. 
Carruba, Clifford; Gabel, Mathew; Hankla, Charles 2008. "Judicial behavior under political constraints: evidence from the European Court of Justice", in American Political Science Review 102, S. 435-452.

Diekmann, Andreas 2005. Empirische Sozialforschung: Grundlagen, Methoden, Anwendungen. 13. Auflage. Hamburg: Rowohlt.

EB 1995. Eurobarometer 44: Expectations for the future. http://ec.europa.eu/public_opinion/ar chives/eb/eb44/chap1_en.htm (Zugriff vom 12.04.2012).

EB 1997. Eurobarometer 47. Die öffentliche Meinung in der EU. http://ec.europa.eu/public_op inion/archives/eb/eb47/eb47_de.pdf (Zugriff vom 12.04.2012).

EB 2001. Special Eurobarometer 150: Highlights Germany. http://ec.europa.eu/public_opinion/ archives/ebs/ebs_150_highlights_en.pdf (Zugriff vom 12.04.2012).

EB 2003. Eurobarometer 60: Die öffentliche Meinung in der EU. http://ec.europa.eu/public_op inion/archives/eb/eb60/eb60_de.pdf (Zugriff vom 12.04.2012).

EB 2004. Eurobarometer 62: Die öffentliche Meinung in der EU. http://ec.europa.eu/public_op inion/archives/eb/eb62/eb_62.de.pdf (Zugriff vom 12.04.2012).

EB 2006 a. Eurobarometer 66: Erste Ergebnisse. http://ec.europa.eu/public_opinion/archives/eb/ eb66/eb66_highlights_de.pdf (Zugriff vom 12.04.2012).

EB 2006 b. Spezial Eurobarometer 251: Die Zukunft Europas. http://ec.europa.eu/public_opini on/archives/ebs/ebs_251_de.pdf (Zugriff vom 12.04.2012).

EB 2007. Eurobarometer zu GALILEO: Die Europäer sind für die Errichtung eines eigenen Navigationssystems durch die EU. Presseerklärung IP/07/764 vom 5. Juni 2007. http://eur opa.eu/rapid/pressReleasesAction.do? reference=IP/07/764\&format=PDF\&aged=1\&langua ge $=$ DE\&guiLanguage $=$ en (Zugriff vom 12.04.2012).

EB 2010 a. Eurobarometer 73: Die öffentliche Meinung in der EU, Teil 1. http://ec.europa.eu/p ublic_opinion/archives/eb/eb73/eb73_vol1_de.pdf (Zugriff vom 12.04.2012).

EB 2010 b. Eurobarometer 73: Die öffentliche Meinung in der EU, Teil 2. http://ec.europa.eu/p ublic_opinion/archives/eb/eb73/eb73_vol2_de.pdf (Zugriff vom 12.04.2012).

EB 2011. Eurobarometer 75: Die öffentliche Meinung in der EU. http://ec.europa.eu/public_op inion/archives/eb/eb75/eb75_de.pdf (Zugriff vom 12.04.2012).

Faulbaum, Frank; Prüfer, Peter; Rexroth, Margrit 2009. Was ist eine gute Frage? Die systematische Evaluation der Fragenqualität. Wiesbaden: VS Verlag für Sozialwissenschaften.

Follesdal, Andreas; Hix, Simon 2006. "Why there is a democratic deficit in the EU. A response to Majone and Moravcsik «, in Journal of Common Market Studies 44, S. 533-562.

Gallup, George H. 1939. Public opinion in a democracy. Princeton: Princeton University Press.

Gramberger, Marc R. 1997. Die Öffentlichkeitsarbeit der Europäischen Kommission 1952-1996: $P R$ zur Legitimation von Integration? Baden-Baden: Nomos.

Haller, Max 2009. Die Europäische Integration als Elitenprozess: Das Ende eines Traums? Wiesbaden: VS Verlag für Sozialwissenschaften.

Hooghe, Liesbet; Marks, Gary 2007. "Sources of euroscepticism«, in Acta Politica 42, S. 119-127.

Hooghe, Liesbet; Marks, Gary 2008. "A postfunctionalist theory of European integration: from permissive consensus to constraining dissensus «, in British Journal of Political Science 39, S. 1-23.

Höpner, Martin; Schäfer, Armin 2010. »A new phase of European integration: organized capitalisms in post-Ricardian Europe", in West European Politics 33, S. 344-368.

Höpner, Martin; Schäfer, Armin 2012. Integration among unequals. How the heterogeneity of European varieties of capitalism shapes the social and democratic deficit of the EU. MPIfG Discussion Paper (im Erscheinen).

Kaase, Max. Hrsg. 1999. Qualitätskriterien der Umfrageforschung. Denkschrift der Deutschen Forschungsgemeinschaft. Berlin: Akademie Verlag.

Karmasin, Matthias; Pitters, Harald 2008. »Methodenprobleme international vergleichender Umfragen am Beispiel des 'Eurobarometer «, in Medien \& Kommunikationsforschung im Vergleich, hrsg. v. Melischek, Gabriele; Seethaler, Josef; Wilke, Jürgen, S. 435-450. Wiesbaden: VS Verlag für Sozialwissenschaften.

Lindberg, Leon N.; Scheingold, Stuart A. 1970. Europe's would-be polity. Patterns of change in the European Community. Englewood Cliffs: Prentice-Hall. 
Moravcsik, Andrew 2008. "The myth of Europe's >democratic deficit«", in Intereconomics. Journal of European Economic Policy 43, S. 331-340.

Neyer, Jürgen 2011. »Wider die Vereinigten Staaten von Europa. Europas demokratische Rechtfertigung ", in Leviathan 39, 4, S. 479-498.

Noelle-Neumann, Elisabeth 1993. "Europa in der öffentlichen Meinung «, in Einstellungen und Lebensbedingungen in Europa, hrsg. v. Glatzer, Wolfgang, S. 11-44. Frankfurt a. M.: Campus.

Oeter, Stefan 2009. »Föderalismus und Demokratie", in Europäisches Verfassungsrecht. Theoretische und dogmatische Grundzüge. 2., vollständig aktualisierte und erweiterte Auflage, hrsg. v. Bogdandy, Armin von; Bast, Jürgen, S. 73-120. Berlin: Springer.

Offe, Claus 2003. Herausforderungen der Demokratie. Zur Integrations- und Leistungsfähigkeit politischer Institutionen. Frankfurt a. M.: Campus.

Pausch, Markus 2008. »Die Eurobarometermacher auf der Zauberinsel: Konstruktion einer europäischen öffentlichen Meinung durch Umfrageforschung «, in SWS Rundschau 48, 3, S. 356-361.

Pausch, Markus 2009. »Eurobarometer und die Konstruktion eines europäischen Bewusstseins «, in Umfrageforschung: Herausforderungen und Grenzen. Österreichische Zeitschrift für Soziologie, Sonderheft 9, hrsg. v. Weichbold, Martin; Bacher, Johann; Wolf, Christof, S. 539-552. Wiesbaden: VS Verlag für Sozialwissenschaften.

Payne, Stanley 1951. The art of asking questions. Princeton: Princeton University Press.

Porst, Rolf 2008. »Question Wording: Zur Formulierung von Fragebogen-Fragen ", in Fragebogen: Ein Arbeitsbuch, S. 95-114. Berlin: Springer.

Schäfer, Armin 2006. »Nach dem permissiven Konsens: Das Demokratiedefizit der Europäischen Union ", in Leviathan 34, 3, S. 350-376.

Scharpf, Fritz W. 1999. Regieren in Europa: Effektiv und demokratisch? Frankfurt a. M.: Campus.

Scharpf, Fritz W. 2009. "Legitimacy in the multilevel European polity «, in European Political Science Review 1, S. 173-204.

Schmitt, Hermann 2003. »The Eurobarometers: their evolution, obvious merits, and ways to add value to them ", in European Union Politics 4, 2, S. 243-251.

Stone Sweet, Alec; Brunell, Thomas 2012. »The European Court of Justice, state noncompliance, and the politics of override", in American Political Science Review 106, S. 204-213.

Strack, Fritz; Martin, Leonard L.; Schwarz, Norbert 1988. »Priming and communication: social determinants of information use in judgments of life satisfaction ", in European Journal of Social Psychology 18, S. 429-442.

Tiemann, Guido; Treib, Oliver; Wimmel, Andreas 2011. Die EU und ibre Bürger. Stuttgart: UTB.

Wallström, Margot 2006. Understanding public opinion. Eröffnungsrede zur EurobarometerKonferenz »Understanding Public Opinion « in Madrid, 27. Oktober 2006. http://europa.e $\mathrm{u} / \mathrm{rapid} /$ pressReleasesAction.do? reference=SPEECH/06/639\&format=PDF\&aged=1\&lang uage $=E N \&$ guiLanguage $=$ en $($ Zugriff vom 12.04.2012).

Wessels, Wolfgang 2008. Das politische System der Europäischen Union. Wiesbaden: VS Verlag für Sozialwissenschaften. 
Zusammenfassung: Das Eurobarometer ist ein wichtiger Datenlieferant der empirischen Integrationsforschung in Europa. Es dient insbesondere der Feststellung des Integrationswillens der europäischen Bürgerinnen und Bürger. Allerdings fungiert die Europäische Kommission selbst als Initiatorin des Eurobarometers. Daraus ergibt sich die Schwierigkeit, dass die Trägerin des Befragungsinstruments den etwaigen demoskopischen Befunden nicht neutral gegenübersteht. Vielmehr ist sie daran interessiert, die Integrationsbereitschaft der Europäerinnen und Europäer so ausgeprägt wie möglich darstellen zu können. Anhand einer Durchsicht der Eurobarometer-Befragungen zwischen 1995 und 2010 wird hier gezeigt, wie die Kommission die Grenze zwischen seriöser Demoskopie und interessengeleiteter Propaganda überschreitet. Diese Überschreitungen betreffen die Informationsüberforderung der Befragten, die einseitige Polung von Fragebatterien, Unterstellungen und Suggestivfragen, nicht ausbalancierte Antwortkategorien, die Hinnahme von Kontexteffekten und tendenziöse Interpretationen integrationspolitisch wichtiger Befunde. Die empirische Europaforschung sollte sich von den Praktiken des Eurobarometers deutlicher als in der Vergangenheit distanzieren.

Stichworte: Europäische Integration, Eurobarometer, Demoskopie, Legitimität

\title{
A critical look at the Eurobarometer. How the Eurobarometer has blurred the line between public opinion research and political propaganda
}

Summary: By reviewing Eurobarometer polls between 1995 and 2010, this article shows how the Commission has blurred the line between serious public opinion research and political propaganda. In order to claim a willingness of the European public for maximum integration, the Eurobarometer has strategically broken the "ten commandments « of public opinion polling. Empirical integration research should distance itself from the Eurobarometer more than in the past.

Keywords: European integration, Eurobarometer, public opinion polls, legitimacy.

\author{
Autoren \\ PD Dr. Martin Höpner \\ Max-Planck-Institut für Gesellschaftsforschung \\ Paulstr. 3 \\ 50676 Köln \\ hoepner@mpifg.de \\ Dipl.-Pol. Bojan Jurczyk \\ Blücherstr. 15 \\ 10961 Berlin \\ Bojan.Jurczyk@gmail.com
}

Leviathan, 40. Jg., 3/2012 https://doi.org/10.5771/0340-0425-2012-3-326 